28

\title{
Designing an inverted light-sheet microscope for long term imaging of organoids
}

Esmée Adegeest, Stefan Semrau

Leiden University, Netherlands

\section{Abstract Text}

Over the past decade, exciting progress in stem cell research has led to the development of organoids as a new system to model human development and disease in vitro. Organoids are three dimensional (3D) cell cultures, derived from primary tissue or stem cells, which recapitulate in vivo biology more closely than conventional cell culture systems. Live fluorescence imaging is an invaluable tool to observe organoid development or their response to perturbations. However, imaging organoids over time remains challenging, due to their large size, demanding culture conditions and light sensitivity. Light-sheet microscopy is ideal to overcome these challenges, due to the restriction of light exposure to the focal plane. Most light-sheet microscope designs, however, require the specimen to be embedded in agarose cylinders or small capillaries, making them unsuitable for organoid systems.

Here, we present the design of a light-sheet microscope that overcomes this limitation. We adopted an inverted microscope setup [1], which we optimized for the use with organoids. In the inverted design of Strnad et al. [1], both the illumination and detection objective face upward and are immersed in water. This configuration allows various 3D cell culture systems to be imaged and cultured in a liquid medium. In their design, however, the illumination of the specimen is limited to one side, which might not be sufficient for organoids that can reach a size of millimeters. In our design we included the option to extend the setup with a second illumination objective opposite to the first. As a result, the orientation of the objectives changed compared to the design of Strnad et al., while keeping the sample holder equally accessible (see Figure 1 ). Furthermore, we also adapted the design of Strnad et al. to enable imaging in the incubator for a long time. To prevent the electronic 
positioners from corrosion, we placed them outside of the incubation chamber. Finally, the combination of a $25 \times 1.1$ NA detection objective and a 15 megapixel camera in our setup allows for highresolution imaging while maintaining a large field of view.

Nevertheless, we found that it is important to carefully design the detection path of the light-sheet microscope in order to minimize vignetting, which becomes apparent when taking images of large specimen. To show the potential of our light-sheet microscope, we will present images of several 3D cell culture systems that are taken with the current design (see Figure 2 for one example). We hope that our insights obtained from building a light-sheet microscope from scratch will be useful for other groups interested in live-cell imaging of organoids.
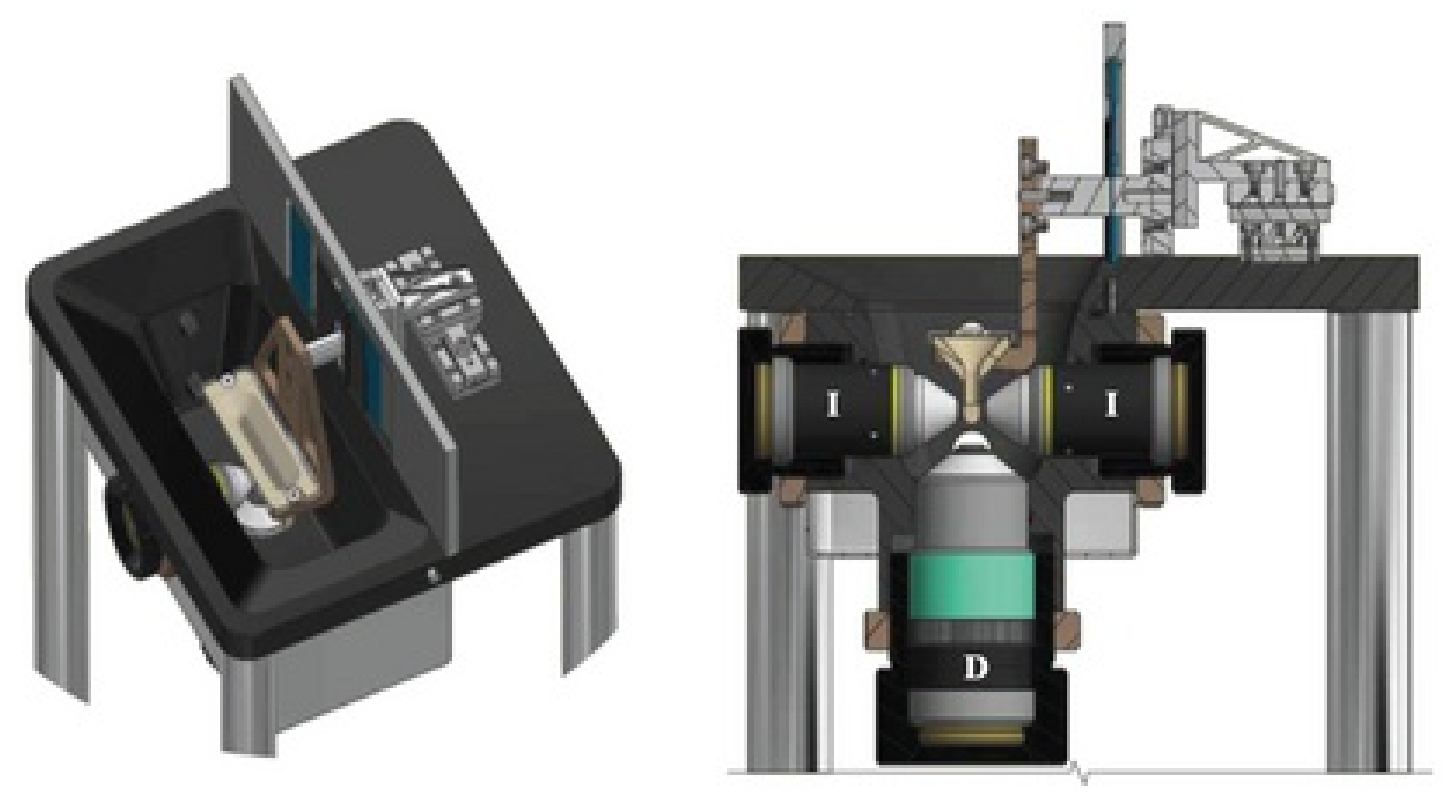

Figure 1. 3D drawings of the objectives mount and sample holder of our light-sheet microscope. The sample holder is placed between two illumination objectives (I) and a detection objective (D) that are immersed in a water reservoir. 


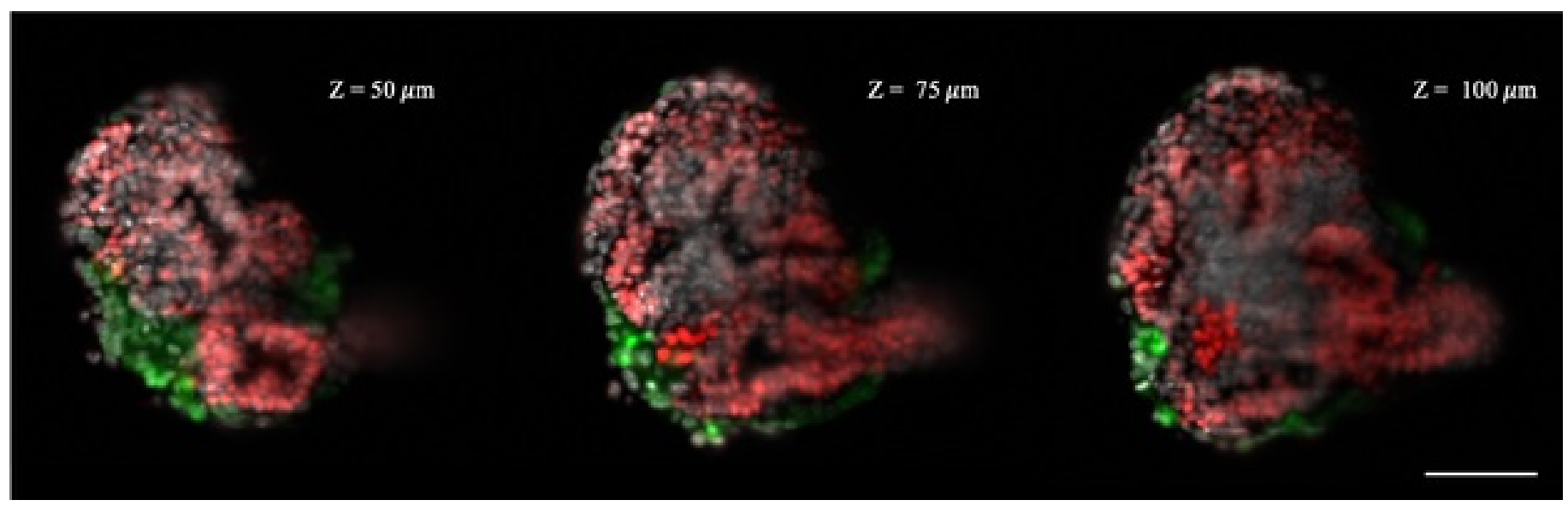

Figure 2. Three Z-planes of an organoid taken with the light-sheet microscope. Different cell types are shown in red and green. All nuclei were stained with DAPI, depicted here in gray. Scale bar: 100 micrometer. 